\title{
ROLE OF CANONICAL WNT-SIGNALLING IN JOINT FORMATION
}

\author{
Daniela Später ${ }^{1}$, Theo P. Hill ${ }^{1}$, Michaela Gruber ${ }^{1,2}$ and Christine Hartmann ${ }^{1}$
}

${ }^{1}$ Institute of Molecular Pathology, Dr. Bohrgasse 7, 1030 Vienna, Austria; ${ }^{2}$ Current address: Department of Cell and Developmental Biology, University of Pennsylvania Cancer Center, 421 Curie Boulevard, Philadelphia, PA19104

\begin{abstract}
The individual elements of the vertebrate skeleton are separated by three different types of joints, fibrous, cartilaginous and synovial joints. Synovial joint formation in the limbs is coupled to the formation of the prechondrogenic condensations, which precede the formation of the joint interzone. We are beginning to understand the signals involved in the formation of prechondrogenic condensations and the subsequent differentiation of cells within the condensations into chondrocytes. However, relatively little is known about the molecules and molecular pathways involved in induction of the early joint interzone and the subsequent formation of the synovial joints. Based on gain-of function studies Wnt-signalling, in particular the canonical pathway, has been implicated in the joint induction process. Here we provide genetic evidence from loss-of function analysis of embryos lacking either the central player of the canonical Wnt-pathway, $\beta$-catenin, in the limb mesenchyme or the two ligands, Wnt9a and Wnt4, demonstrating that canonical Wnt-signalling plays an important role in suppressing the chondrogenic potential of cells in the joint thereby actively allowing joint formation. Furthermore our data show that the $\beta$-catenin activity is not essential for the induction of molecular markers expressed in the joint interzone. Thus, suggesting that canonical Wnt-signalling is not required for the induction, but for the subsequent maintenance of the fate of the joint interzone cells.
\end{abstract}

Key words: Wnt, joint, synovial chondroid metaplasia, $\beta$ catenin

Address for correspondence:

Christine Hartmann,

Institute of Molecular Pathology,

Dr. Bohrgasse 7,

1030 Vienna, Austria

Phone: (+43)-1-79730-840

Fax: (+43)-1-7987153

E-mail: hartmann@imp.univie.ac.at

\section{Introduction}

The formation of synovial joints in the appendicular skeleton is a multistep process, which is tightly connected to the formation of the prechondrogenic anlagen. The latter arise by condensation of mesenchymal cells at the sites of the future elements. In the case of the appendicular skeleton, the early condensations can form branched structures, like in the forelimb where the humerus branches into radius and ulna. In contrast, the carpal elements in the wrist arise as separate condensations (Archer et al., 2003). The induction of a joint occurs at the presumptive joint location within the branched condensations. The location of the future joint is probably determined by a combination of genes involved in patterning of the limb. The induction process involves changes in the cell shape from round to more flattened, elongated cells and the induction of genes more or less specific for the early interzone, among others Gdf5, Wnt4, Wnt9a (formerly known as Wnt14), Gli1, Gli3, CD44, autotaxin, and chordin (Francis-West et al., 1999a; Guo et al., 2004; Hartmann and Tabin, 2001). The expression of these markers precedes or coincides with the downregulation of type II Collagen (Col2 $\alpha 1)$ and Sox9. The down-regulation of these chondrogenic markers during joint formation suggested that an essential step in joint formation is to block the further differentiation of these cells into chondrocytes. This notion is also supported by the finding that mice lacking noggin, which acts as an antagonist of the activity of Bone morphogenic proteins (BMP), have no signs of joint formation (Brunet et al., 1998; Tylzanowski et al., 2006). Noggin, is expressed initially throughout the prechondrogenic region including the joint interzone, and becomes later upregulated at the distal ends of the skeletal elements adjacent to the joints. Recent studies in the chicken have shown that retroviral over expression of Wnt9a (formerly known as Wnt14), one of the 19 ligands of the Wnt-signalling pathway results in a blockage of chondrogenic differentation of the infected prechondrogenic region, and morphological and molecular changes resembling the joint interzone (Hartmann and Tabin, 2001). Wnt9a is expressed in the early joint interzone in chick and mouse. Phenotypic changes similar to those observed in chicken have also been seen in the mouse using a transgenic approach, whereby Wnt9a was misexpressed under the control of the Col2 $\alpha 1$ promoter (Guo et al., 2004). The mouse gainof function studies by Guo et al. (2004) suggested, in addition, that Wnt9a could be signalling through the canonical Wnt-pathway. This pathway is activated by the binding of a ligand to a receptor complex composed out of a member of the Frizzled family of serpentine receptors and one of the LRP5/6 co-receptors leading to an 
intracellular stabilization of the key component, $\beta$-catenin. Upon stabilization, $\beta$-catenin is translocated into the nucleus where it interacts with transcription factors of the $\mathrm{TCF} /$ Lef family and activates transcription of various target genes (Logan and Nusse, 2004; Wnt-homepage: http:// www.stanford.edu/ rnusse/wntwindow.html). Together the misexpression experiments performed in chicken and mouse suggested that Wnt9a-signalling mediated by $\beta$ catenin is involved in joint induction. Furthermore, Guo and colleagues showed that loss of $\beta$-catenin in Col $2 \alpha 1$ expressing cells results in fusion of some tarsal joints observed at E15.5 (Guo et al., 2004). Based on the studies in mouse, it was postulated that canonical Wnt-signaling is required for the induction of Gdf5 in the joint interzone, possibly acting as the joint inducer (Guo et al., 2004).

In order to address whether signaling by Wnt9a is indeed necessary for joint formation we had generated mice lacking Wnt9a activity and performed morphological, histological and marker analysis focusing on the joints of these animals. Since at least two other Wnt-genes, Wnt4 and Wnt16, are expressed in the joints we also generated double mutants for Wnt9a and Wnt4. In addition, we reexamined the joint phenotypes in mice lacking $\beta$-catenin in the entire limb mesenchyme (Später et al., 2006). Here we show a more extensive marker analysis supporting our previously published loss-of function analysis of the Wnt mutants, which suggested that Wnt9a and Wnt4 act redundantly in joint maintenance actively repressing the chondrogenic potential of synovial and fibrous joint cells, but that they are probably not required for the formation of the early joint interzone. This is supported by our accompanying analysis of limbs lacking mesenchymal $\beta$ catenin activity at early stages, when the interzone is being formed, which revealed that early joint markers, in particularly $G d f 5$ are still expressed, suggesting that $G d f 5$ expression is not dependent on canonical Wnt-signalling.

\section{Material and Methods}

\section{Mouse mutants}

Wnt9a and Wnt4 mutants were genotyped by PCR (Später et al., 2006; Stark et al., 1994). Mice lacking $\beta$-catenin activity in the limb mesenchyme were generated as previously described (Hill et al., 2005).

\section{Histology and in situ hybridizations}

For histology and non-radioactive section in situ hybridization, tissue was treated as previously described (Hill et al., 2005).

\section{Retroviral work, micromass and chondrocyte cultures}

The RCAS-AP, RCAS-Wnt5a, RCAS-Wnt9a, RCASWnt4, RCAS-Wnt3a and RCAS- $\Delta \mathrm{N} \beta$-cat viruses were previously described (Hartmann and Tabin, 2000; Hartmann and Tabin, 2001; Kengaku et al., 1998). Micromass cultures using chicken HH22-23 limb buds were performed as previously described (Hartmann and Tabin, 2001), cartilage nodule formation was visualized at day 4 by Alcian blue staining. Chondrocytes isolated from the caudal part of day 18 chick sternae (Koyama et al., 1999) were cultured for one day, collected and plated at $5 \times 10^{5}$ cells/well density in a six well plate. The following day chondrocytes were infected using $5 \mu \mathrm{l}$ viral supernatant per well (titers: $6-8 \times 10^{8} \mathrm{pfu} / \mathrm{ml}$ ) and cultured for 3-4 days in DMEM:F12 (Invitrogen). All experiments were done in triplicates.

\section{Western blot analysis}

$50 \mu \mathrm{g}$ of protein extract, isolated from cultured chicken sternal chondrocytes infected with different RCAS viruses, were loaded per lane. Luminal detection was performed using an antibody against chicken $\beta$-catenin (1:800, Sigma C7027), followed by incubation with a HRP-conjugated secondary antibody (1:2500; Promega).

\section{Immunohistochemistry}

For immunohistochemical staining of cultured chondrocytes, cells were fixed for $15 \mathrm{~min}$ at RT with $4 \%$ paraformaldehyde in PBS, washed twice with PBS. Endogenous peroxidase activity was inactivated by $30 \mathrm{~min}$ incubation in $1 \% \mathrm{H}_{2} \mathrm{O}_{2}$ in PBS. Cells were subsequently washed three times with PBS, blocked for $30 \mathrm{~min}$ with PBS; $10 \%$ FCS, $0.1 \%$ Triton-X100, incubated with primary antibodies against Collagen type II (II-II6B3 supernatant, 1:30) or Collagen type III (3B2 supernatant 1:30) from the Developmental Hybridoma Bank (Iowa). Signaldetection was done using a biotinylated anti-mouse secondary antibody (dilution 1 in 250; Vector labs) in combination with the ABC kit (Vector labs) and DAB (Sigma) as a substrate.

\section{Results}

Mice lacking Wnt9a activity displayed a shortening of the proximal long bones and mild joint abnormalities at birth (Später et al, 2006). The most severely affected joint was the humeral-radial joint (HRJ) in the elbow region, in which synovial cells differentiated in $100 \%$ of the mutants into an ectopic cartilage element (Figure 1A,B). This phenotype is known in humans as synovial chondroid metaplasia. We examined earlier developmental stages to determine the onset of the phenotype. No obvious differences were evident in the expression of Col $2 \alpha 1$, Gdf5, Gli3 and Wnt4 in the elbow joints from Wnt9 $a^{-/}$and wildtype embryos at E13.5 (Figure 1E-H). The first signs of ectopic cartilage formation in the HRJ were observed at E15.5, visible as a small ectopic cluster of Col $2 \alpha 1$ positive cells and a broad ectopic Sox9 domain in the outer joint region (Figure $1 C^{\prime}, D^{\prime}$ ). In addition, partial fusions of the carpal elements $\mathrm{c} / 3$ and of the intermediate cuneiform and navicular tarsal elements were observed in approx. 80\% of the mutants at birth (Später et al., 2006). Not only synovial joints were affected in the Wnt9a mutants, ectopic cartilage formation was also observed in the sutures, which are fibrous joints separating the bony plates in the skull (Später et al., 2006).

As only a few synovial joints were affected in the Wnt9a mutants and since two other Wnt-genes, Wnt4 and Wnt16, are known to be expressed overlapping with Wnt9a 

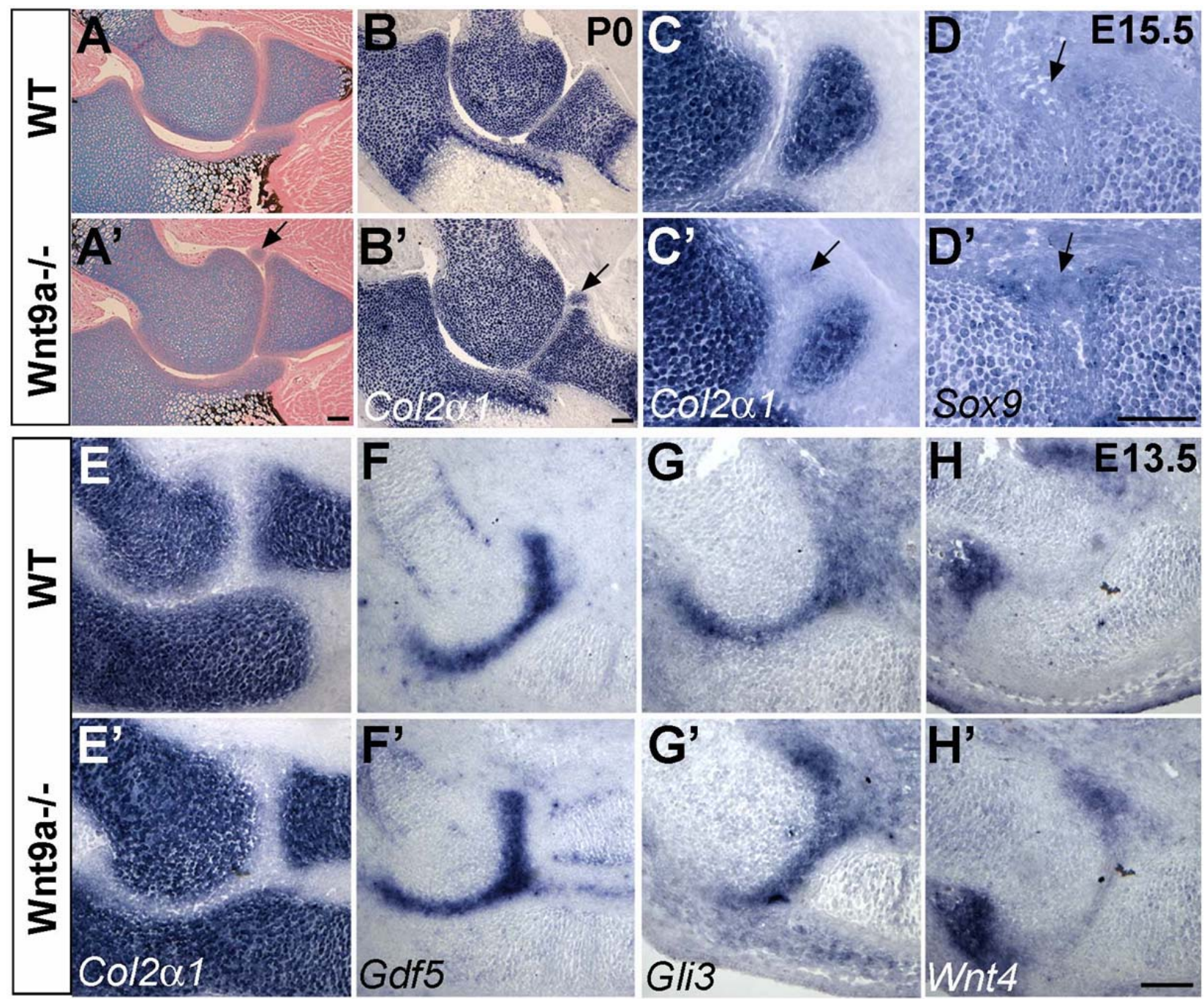

Figure 1. Synovial chondroid metaplasia in the humeral-radial joint (HRJ) of Wnt9a mutants. (A-B') Sections through newborn wild-type and Wnt9a mutant forelimbs: normal HRJ in (A, B) ectopic cartilage nodule visualized by Alcian blue staining (arrow in A') and in situ hybridization for Col2 $\alpha 1$ transcripts (arrow in B'). (C-D') In situ hybridizations on sections through E15.5 wild-type and Wnt9a mutant forelimbs: (C) no Col2 $\alpha 1$ staining in the wild-type, (C") ectopic Col2 $\alpha 1$ expression in the HRJ (arrow). (D) No Sox9 expression in the wild-type HRJ, (D') ectopic expression of Sox9 in the mutant (arrow). (E-H') In situ hybridizations on sections through E13.5 wildtype and Wnt9a mutant forelimbs: no difference in the expression patterns of Col2 $\alpha 1$ (E, E'), Gdf5 (F, F'), Gli3 $\left(\mathrm{G}, \mathrm{G}^{\prime}\right)$, Wht4 (H, H') between wild-type and mutant. Scale bars are $100 \mu \mathrm{m}$.

in the joints we generated double mutants for Wnt9a and Wnt4 to test for possible functional redundancy. Notably Wnt4 mutants have no reported skeletal genotypes (Stark et al., 1994). Wnt9a and Wnt4 mutants displayed synovial chondroid metaplasia in two additional major joints, the knee and the ankle joint (Figure 2A-B'). In addition, 100\% of the double mutant newborns displayed complete fusions of carpal elements 2, c, and 3 (Figure 2C-C"), which resulted in an abnormal flexure of the forelimb autopod. Closer examination of the articular regions revealed that $\mathrm{Wnt}^{-/-}$; Wnt9 $\mathrm{a}^{-/}$articular chondrocytes are stained more intensely by alcian blue, when compared to wild-type, suggesting that their extracellular matrix was altered (Figure 2D-E'). The nature of this articular chondrocyte phenotype will be examined in the future using molecular markers.

The onset of the fusion phenotype of the carpal elements observed in newborns was analyzed further at earlier stages using a variety of molecular markers. This analysis revealed that the carpal elements were not yet fused in the double mutants at E13.5 (Figure $3 \mathrm{~A}$ ), but they were fused at E15.5 (Figure 3B). In the foot the intermediate cuneiform and navicular, as well as the calcaneus and cuboid tarsal elements were fused in newborns (Figure 4B', B'). Occasionally we observed the formation of an ectopic cartilage piece in ligaments of the digits (asterisk in Figure 4B"). Similarly to the situation in the carpal region individual tarsal elements could be identified by Col $2 \alpha 1$ staining as separate condensations, surrounded by cells expressing Gdf5 at E13.5 (Figure 4C', D'), but were fused at E15.5 (data not shown).

The phenotype of the double mutants suggested that early Wnt-signalling is not necessary for the formation of the joint interzone. However, in addition to Wnt9a and Wnt4, Wnt16 is also expressed in the joint region and could, in principle, still compensate for the loss of the other two 

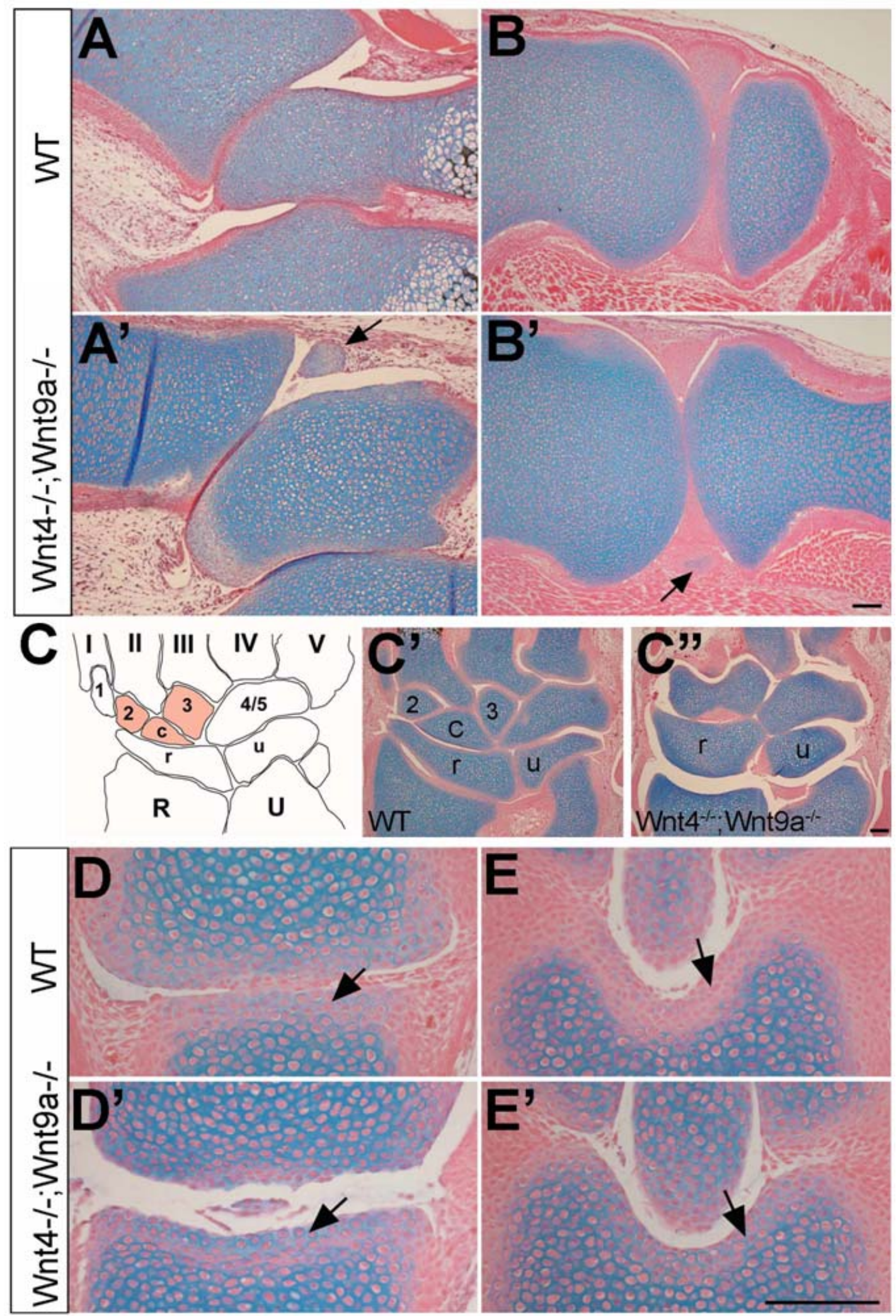

Figure 2. Joint alterations in newborn Wnt4;Wnt9a double mutant mice. (A-E') Alcian blue staining on sections through the ankle, knee, wrist and digit regions of newborn wild-type and Wnt4-/-;Wnt9a-/- mice. (A) Normal ankle joint. (A') Ectopic cartilage nodule instead of the synovial tissue in the double mutants (arrow). (B) Normal knee joint. (B') Ectopic cartilage nodule in the fibrous tissue of the knee capsule (arrow). (C) Schematic diagram showing the arrangement of carpal elements, with carpals 2, c, 3 highlighted in red. (C') Normal arrangement of carpals in the wild-type. (C") Fusion of carpals 2, c, 3 in the double mutants. R: radius; U: ulna; I-V: digits, r: radial element; u: ulnar element. (D, E) Sections through a normal metacarpal-phalangeal joint showing low intensity alcian blue staining in the articular cell layers (arrows). (D', E') Sections through a mutant metacarpalphalangeal joint showing more intense alcian blue staining in the articular cell layers compared to wild-type (arrows). Scale bars are $100 \mu \mathrm{m}$. 


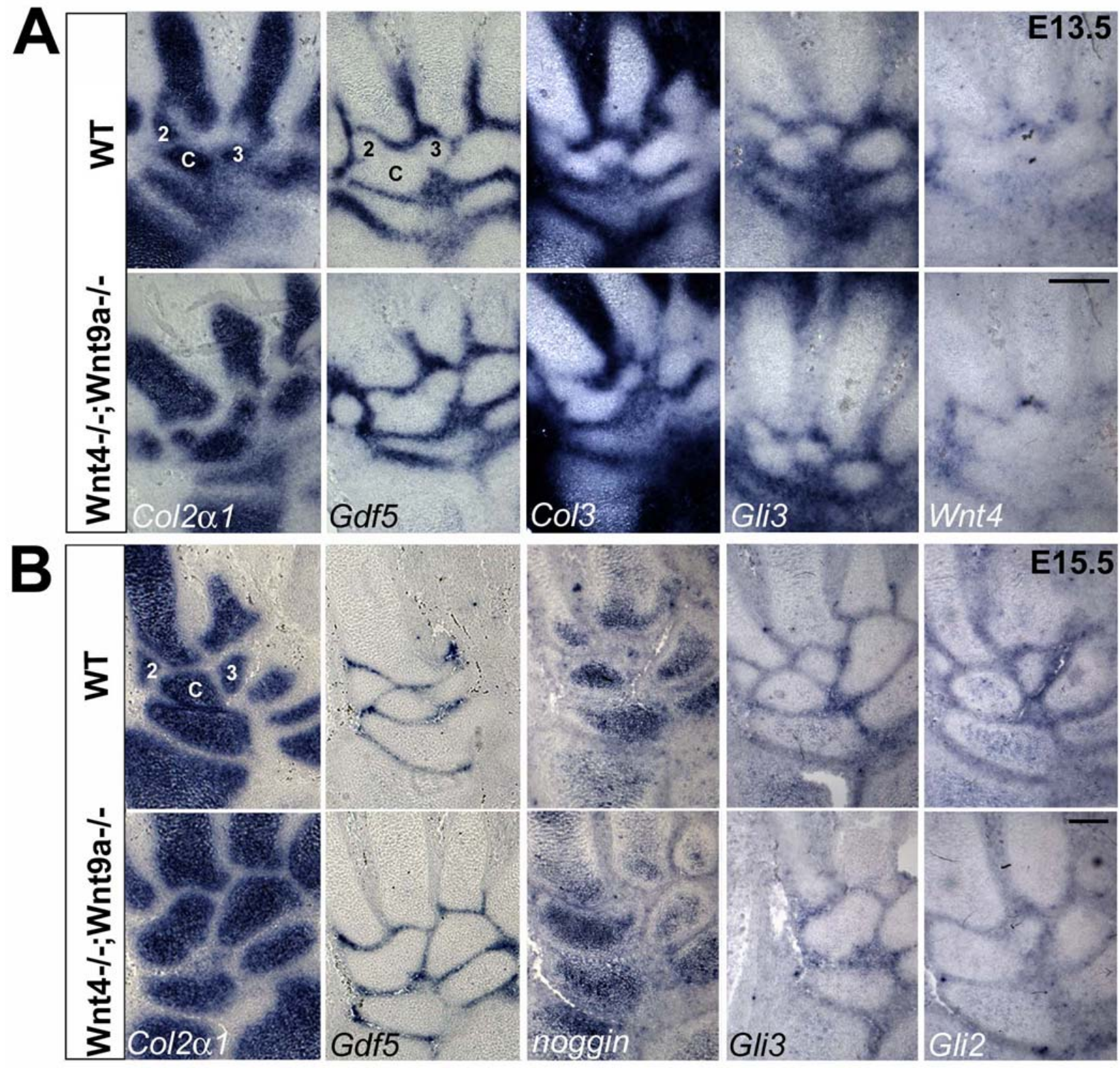

Figure 3. Comparison of molecular marker expression between wild-type and Wnt4;Wnt9a double mutants during carpal development. (A) In situ hybridizations on sections through the carpal region of wild-type (upper panel) and Wnt4-/-;Wnt9a-/- embryos (lower panel) at E13.5. No difference is visible in the expression patterns of the markers: Col2 $\alpha 1$, Gdf5, Col3, Gli3, Wnt4. (B) In situ hybridizations on sections through the region of the carpals of wildtype (upper panel) and Wnt4-/-;Wnt9a-/- embryos (lower panel) at E15.5. No difference is visible in the expression patterns of the markers: Col2 $\alpha 1$, Gdf5, noggin, Gli3, Gli2. Scale bars are $100 \mu \mathrm{m}$.

Wnt-genes. Retroviral mediated misexpression of Wnt4 and Wnt9a both inhibited chondrogenesis in chick micromass cultures, as did ectopic expression of a stabilized form of $\beta$-catenin ( $\Delta \mathrm{N} \beta$-cat) (Figure $5 \mathrm{~B}-\mathrm{D})$ see also (Guo et al., 2004; Hartmann and Tabin, 2001), suggesting that both Wnts are probably signalling through the $\beta$-catenin pathway (Guo et al., 2004; Hartmann and Tabin, 2000). Therefore, we asked the question, if the formation of the early joint interzone is affected in mice lacking mesenchymal $\beta$-catenin activity in the limbs $(\beta$ -

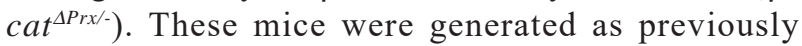
described (Hill et al., 2005). The early condensations of the skeletal elements visualized by Col $2 \alpha 1$ in situ hybridization are in principle formed, but are slightly abnormal, since a part of the scapula anlage is missing and the digits are not formed normally (Hill et al., 2006) (Figure 6A'). Analysis of early joint interzone markers such as Gdf5, Wnt4 and Gli1 revealed that all three markers were still expressed in the prospective joint region (Figure 6B'-D'), although their expression domains were somewhat broader compared to heterozygous littermates (Figure 6B-D). In wild-type limbs Col2 $\alpha 1$ expression starts to be down-regulated in the joint region around E12.5 (arrowhead in Figure 6E), however, this down-regulation was not clearly visible in $\beta$-cat ${ }^{\Delta \text { Prx/- }}$ mice (arrowheads in Figure 6E'). At E12.5 Gdf5 expression was similarly expressed comparing the joints of wild-type and mutant limbs (Figure 6F, F'). In contrast to the broadened Gdf5 


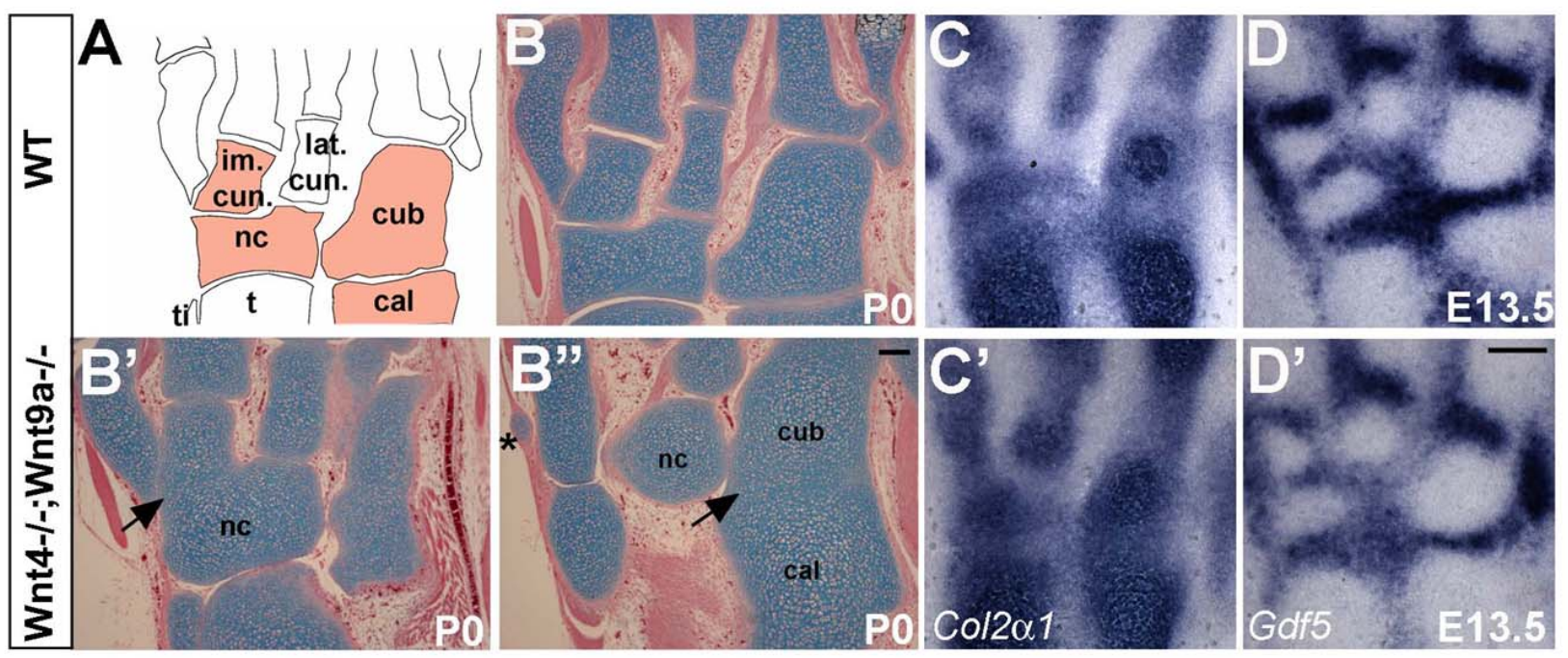

Figure 4. Joint fusions between tarsal elements in Wnt4;Wnt9a double mutants. (A-B') Sections through the tarsal region of wild-type and Wnt4-/-;Wnt9a-/- newborns. (A) Schematic diagram showing the arrangement of tarsal elements: cal: calcaneus; cub: cuboid; 1.c.: lateral cuneiform; i.c.: intermediate cuneiform; nc: navicular; $t$ : tarsal. (B) Alcian blue staining of a wild-type tarsal region. (B', B') Alcian blue staining of a Wnt4-/-;Wnt9a-/mutant showing fusion between the intermediate cuneiform and navicular tarsal elements (arrow in B") and between the calcaneus and cuboid tarsal elements (arrow in B") and synovial chondroid metaplasia in the joint capsule ligament of digit I (asterisk in B"). Pattern of condensation of tarsal elements visualized by Col $2 \alpha 1$ expression is similar between wild-type (C) and mutant (C'). Gdf5 expression pattern is similar between wild-type (D) and mutant (D'). Scale bars are $100 \mu \mathrm{m}$.

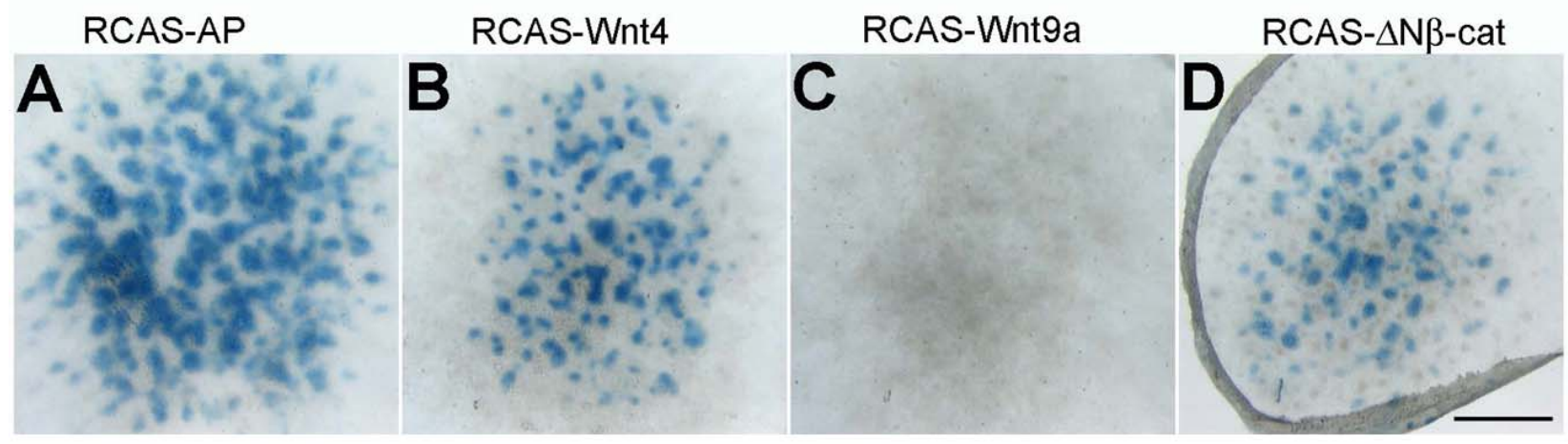

Figure 5. Inhibitory effects of Wnt4, Wnt9a and stabilized $\beta$-cat on cartilage formation. (A-D) Alcian blue staining visualizing cartilage nodule formation in micromass cultures at day 4. (A) RCAS-AP control infected cultures. (B) RCAS-Wnt4 infected cultures show a reduced number of cartilage nodules. (C) RCAS-Wnt9a infected cultures show a complete block in cartilage nodule formation. (D) RCAS- $\Delta \mathrm{N}-\beta$-catenin show a reduced number of alcian blue stained cartilage nodules. Scale bar is $1 \mathrm{~mm}$.

domain in the mutant at E11.5, the Gdf5 expression domain was much more refined at E12.5, but the expression level was slightly reduced compared to the wild-type (Figure $\left.6 F^{\prime}\right)$. At E14.5, when Col2 $\alpha 1$ expression is normally absent from the wild-type joint (Figure 6G), Col $2 \alpha 1$ was still expressed in the "joint-like" region of the mutant, but at slightly lower levels compared to the neighbouring cartilage element (arrowheads in Figure 6G'). Gdf5 was like in the wild-type at E14.5 still expressed in the mutant, but more restricted to the lateral edges (arrowheads Figure $\left.6 \mathrm{H}^{\prime}\right)$. The presence of the early joint markers suggests that the process of joint induction is not affected by the loss of $\beta$-catenin. On the other hand, what seems to be affected is the down-regulation of Col2 $\alpha 1$ expression and suppression of chondrogenesis in the joint region fails.
Since certain Wnts can interfere with chondrogenesis in micromass cultures (see above) we wanted to address the effect of ectopic activation of Wnt-signalling on proliferating chondrocytes. For that reason, chondrocytes isolated from the caudal part of day 18 chick sternae were infected with retroviruses expressing either alkaline phosphatase (AP), Wnt5a, Wnt9a, Wnt3a or the stabilized form of $\beta$-catenin. Chondrocytes were cultured for 4 days and stained for Collagen II and Collagen III. While control infected chondrocytes (RCAS-AP) and RCAS-Wnt5a infected chondrocytes still produced a Collagen II positive matrix and retained a cuboidal shape, chondrocytes infected either with RCAS-Wnt9a, RCAS-Wnt3a or RCAS- $\Delta N \beta$-cat down-regulated Collagen II production. Furthermore, the morphology of the cells in RCAS-Wnt9a, 

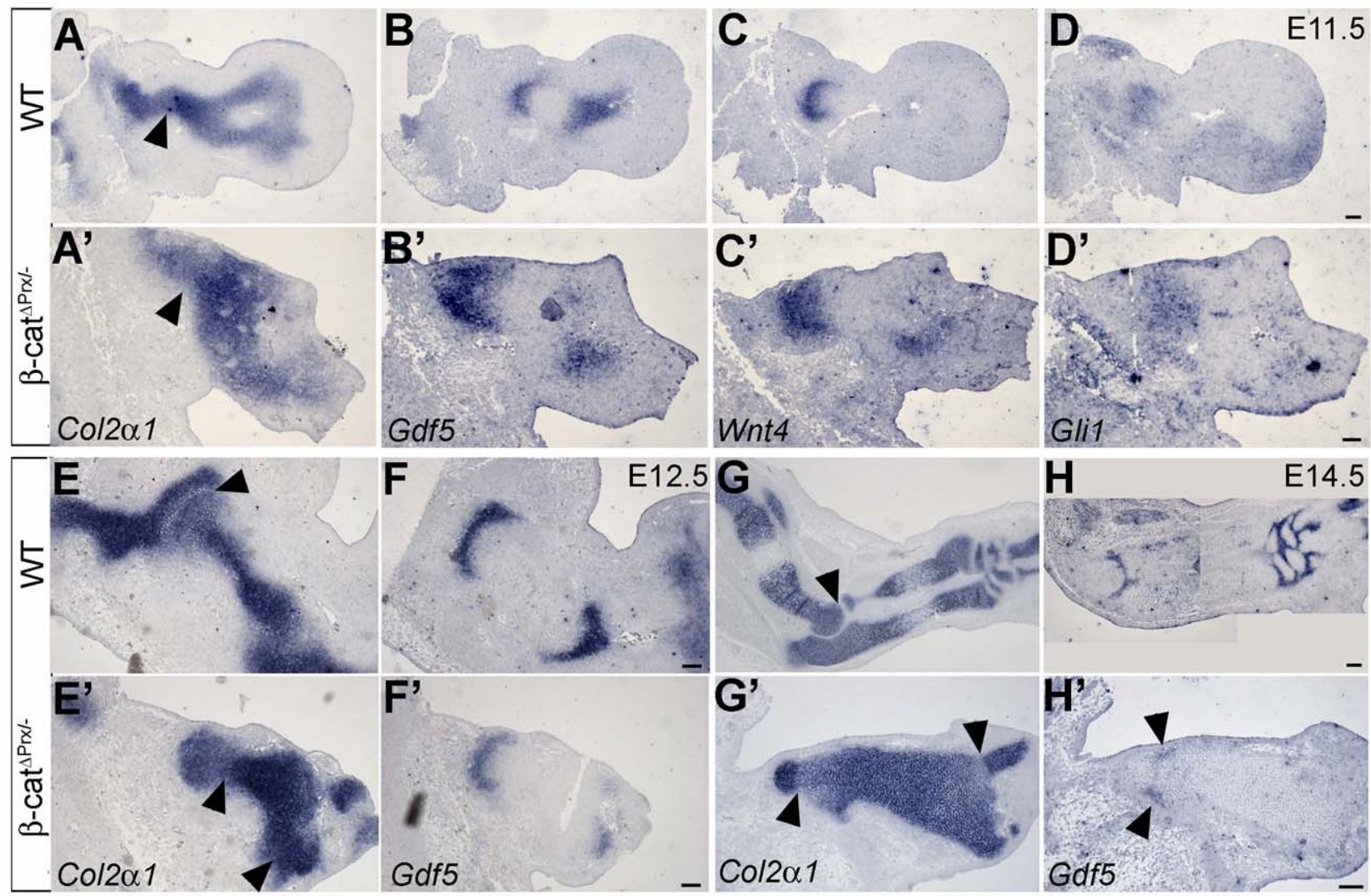

Figure 6. Analysis of joint induction and formation in mouse limbs lacking mesenchymal $\beta$-catenin $\left(\beta\right.$-cat $\left.{ }^{\Delta \mathrm{Prx} /}\right)$ activity. (A-H') Marker analysis by in situ hybridization of wild-type $(\mathrm{A}-\mathrm{H})$ and $\beta$-cat ${ }^{\Delta \mathrm{Pr} /-}$ mutant $\left(\mathrm{A}^{\prime}\right.$ - $\left.\mathrm{H}^{\prime}\right)$ forelimbs at E11.5 (A-D'), E12.5 (E-F') and E14.5 (G-H'). (A) Col2 $\alpha 1$ expression showing prechondrogenic condensations of the scapula, humerus, radius and ulna (from left to right; arrowhead marks the position of the joint between the scapula and humerus) in wild-type (A) and mutant (A'). Gdf5 expression in the shoulder joint is similar between the wild-type (B) and the mutant (B'); note: Gdf5 is also expressed in elbow joints as well as the space between radius and ulna in the section of the wild-type limb (B). (B') Wnt4 expression in the shoulder joint is similar between wildtype (C) and mutant (C'). Gli1 expression is similar between wild-type (D) and mutant (D'). Note that in the mutant the expression domain of the early joint interzone markers, Gdf5, Wnt4 and Gli1 is broader compared to the wildtype. (E) Down-regulation of Col2 $\alpha 1$ expression in the shoulder joint in the wildtype (arrowhead). (E') Incomplete down-regulation of Col2 $\alpha 1$ expression in joints of the mutant (arrowheads). (F) Gdf5 expression in the wild-type joints. (F') Gdf5 is still expressed in the joints of the mutant. (G) Complete absence of Col2 $\alpha 1$ expression in the wild-type joints (see arrowhead in elbow joint). (G') Incomplete down-regulation of Col2 $\alpha 1$ expression in mutant joint-like areas (arrowheads). (H) Gdf5 expression in wild-type joints. (H') Weak remaining Gdf5 expression in joint-like area of the mutant (arrowheads). Scale bars are $100 \mu \mathrm{m}$.

Wnt3a and $\Delta \mathrm{N} \beta$-cat infected cultures changed to a more fibroblast-like appearance and the cells produced a Collagen III rich matrix (Figure 7A). Interestingly, western blot analysis revealed that infection of chondrocytes with RCAS viruses expressing either the canonical Wnt-ligand, Wnt3a, or Wnt9a resulted in increased levels of $\beta$-catenin protein, in contrast to RCAS-AP control infected or cultures infected with an RCAS-virus expressing the noncanonical Wnt-lignad, Wnt5a (Figure 7B). Thus, the phenotypic changes in the chondrocyte cultures infected with RCAS-Wnt9a or Wnt3a are probably due to the increase in $\beta$-catenin levels. Particularly, since this effect can be mimicked by infecting the cells with an RCAS expressing a stable form of $\beta$-catenin.

\section{Discussion}

All joints are initially formed normally in double mutants for Wnt9a and Wnt4, this suggests that those two Wnt genes are not required for the initiation of joint formation. However, around E15.5 cells in the joint region of various joints seem to fail to maintain their joint cell identity in the absence of these two ligands. Therefore, we propose that Wnt-signalling, probably through the canonical Wntpathway, is actively involved in maintaining joint identity by repressing the chondrogenic potential of cells within the joint region. Some of these cells might still remain as precursor cells, which are still bipotential, meaning that they can give rise to chondrocytes as well as synoviocytes or joint capsule cells. This hypothesis is supported by previous findings showing that synovial tissue contains chondro-progenitors (Nalin et al., 1995; Nishimura et al., 
A
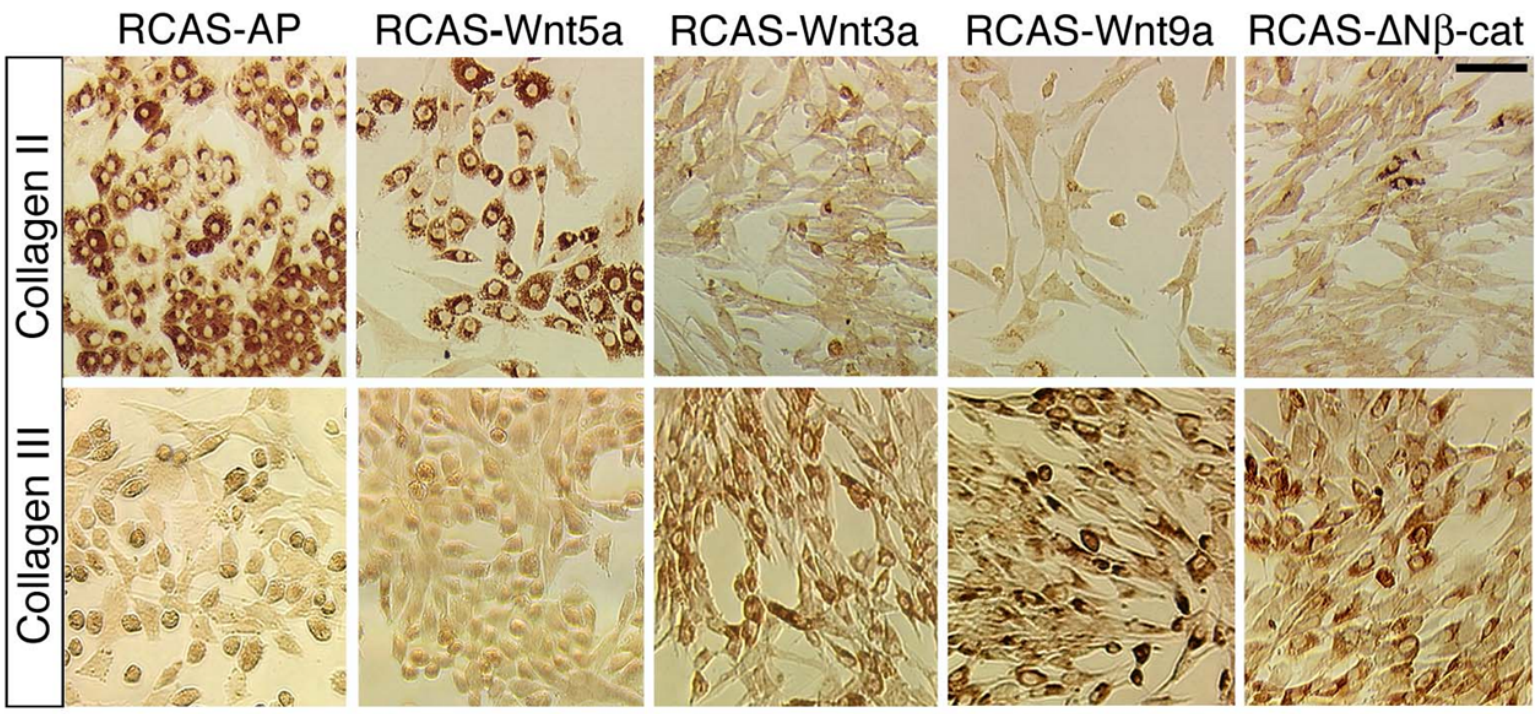

B

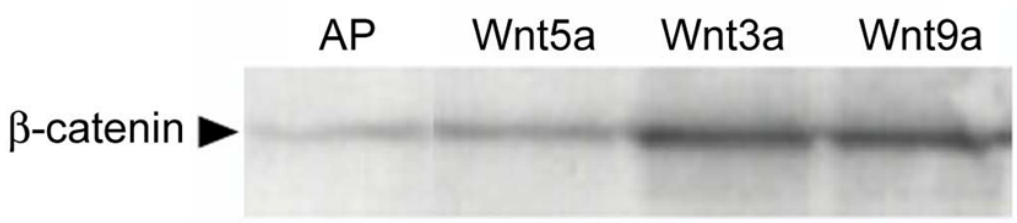

Figure 7. Changes in cultured chondrocytes upon retroviral misexpression of various Wnts and stabilized $\beta$ catenin. (A) Immunohistochemical staining for extracellular matrix proteins Collagen II and Collagen III on chondrocytes infected with RCAS-AP (control), RCAS-Wnt5a (non-canonical Wnt), RCAS-Wnt3a (canonical Wnt), RCAS-Wnt9a and RCAS- $\Delta \mathrm{N} \beta$-cat. Collagen II is not down-regulated in Wnt5a infected cells, but is downregulated in RCAS-Wnt3a, Wnt9a and $\triangle \mathrm{N} \beta$-cat infected cells. RCAS-AP and Wnt5a infected cells do not stain positive for Collagen III, while RCAS-Wnt3a, Wnt9a and $\Delta \mathrm{N} \beta$-cat infected cells do. (B) Western-blot showing increased $\beta$-catenin protein levels in chondrocytes infected with RCAS-Wnt3a and Wnt9a. Scale bars are $20 \mu \mathrm{m}$.

1999). Not all of the joints are affected in the Wnt9a; Wnt4 double mutants. This could be due to the fact that a third Wnt, Wnt16, is expressed in the joints as well (Guo et al., 2004). Therefore, it remains possible that Wnts might play a more important role in joint initiation and formation than what we propose on the basis of the analysis of the double mutants. However, our molecular marker analysis in the limbs, which lack $\beta$-catenin activity in the mesenchyme, demonstrated that the joint interzone markers Gdf5, Wnt4 and Gli1 are initially expressed (E11.5) and remain to be expressed even at later stages. These observations favour our hypothesis that canonical Wnt signalling is not required for the initiation of joints and also show that it is not required for $G d f 5$ expression as previously proposed (Guo et al., 2004). Furthermore, we never observed ectopic activation of $G d f 5$ expression in micromass cultures infected either with Wnt9a, Wnt4 or $\Delta \mathrm{N}$ - $\beta$-catenin expressing retroviruses (C. Hartmann, unpublished observation). Thus, in analogy to the previously identified role of $\beta$-catenin in osteo-chondroprogenitors (Day et al., 2005; Hill et al., 2005) the canonical Wnt-pathway is likely to be required to repress the chondrogenic potential of what might be a synovio-chondroprogenitor. This strong antichondrogenic activity of the canonical Wnt-signalling pathway is also reflected by the observation that increased levels of $\beta$-catenin can revert chondrocytes in vitro into more fibroblast-like cells expressing different extracellular matrix molecules. What the nature of these cells is remains to be determined in the future.

In conclusion the canonical Wnt-pathway seems to be important to allow the differentiation of the joint lineage by actively suppressing the chondrogenic potential in precursor cells (see Model Figure 8). The data presented here together with the previously identified role of $\beta$ catenin in the osteoblast lineage (Day et al., 2005, Hill et al., 2005, Rodda and McMahon, 2006) suggests that during skeletal lineage differentiation, chondrogenesis might be the default state and that inhibition of chondrogenesis by a $\beta$-catenin mediated Wnt-signal is required for differentiation of the other two cell lineages. While the Wnt signals involved in osteoblast lineage differentiation remain to be identified, our data suggest that Wnt9a together with Wnt4 and probably Wnt16 are the signals essential during joint lineage formation.

From a medical point of view, the observed synovial chondroid metaplasia phenotype in the elbow in case of loss-of Wnt9a activity alone or in the knee and ankle joints in mutants lacking both Wnt9a and Wnt4 activity is very interesting. Similar pathological alterations of the joint with spontaneous formation of cartilaginous nodules are known in humans as well. In their more severe form they are referred to as synovial chondromatosis. The etiology of 


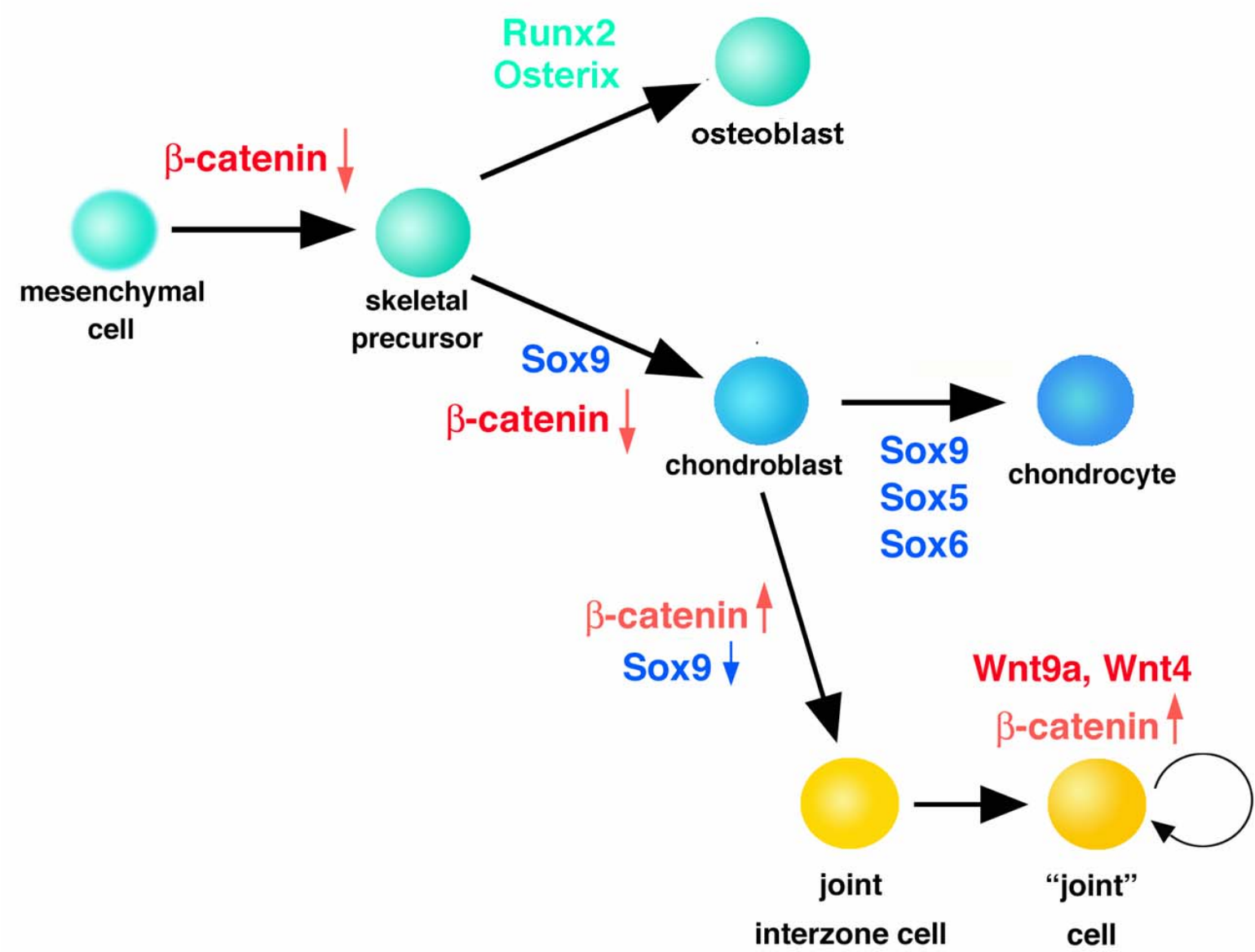

Figure 8. Model for the requirement of canonical Wnt-signalling during joint lineage differentiation. Low levels of $\beta$-catenin are required for cells to enter the skeletal lineage. For the further differentiation of skeletal precursors into chondroblasts / chondrocytes $\beta$-catenin levels need to be reduced. For the establishment of a true joint interzone $\beta$-catenin levels need to be elevated to suppress the chondrogenic potential of interzone cells and levels of $\beta$-catenin need to stay elevated in joint cells to maintain joint integrity.

this disease is still largely unknown. Therefore, it will be interesting to examine whether the canonical Wntsignalling pathway is in some way altered in affected individuals.

\section{Acknowledgements}

We thank Cliff Tabin for his support and generosity; the Developmental Hybridoma Bank (Iowa) for the Collagen II and Collagen III antibodies. This work was supported by Boehringer Ingelheim and the EU (NoE Cells into Organs).

\section{References}

Archer CW, Dowthwaite GP, Francis-West P (2003) Development of synovial joints. Birth Defects Res C Embryo Today 69: 144-155.

Brunet LJ, McMahon, JA, McMahon AP, Harland RM (1998) Noggin, cartilage morphogenesis, and joint formation in the mammalian skeleton. Science 280: 1455 1457.
Day TF, Guo X, Garrett-Beal L, Yang Y (2005) Wnt/ $\beta$-Catenin Signaling in Mesenchymal Progenitors Controls Osteoblast and Chondrocyte Differentiation during Vertebrate Skeletogenesis. Dev Cell 8: 739-750.

Francis-West PH, Parish J, Lee K, Archer CW (1999a) BMP/GDF-signalling interactions during synovial joint development. Cell Tissue Res 296: 111-119.

Guo X, Day TF, Jiang X, Garrett-Beal L, Topol L, Yang $Y$ (2004) Wnt/ $\beta$-catenin signaling is sufficient and necessary for synovial joint formation. Genes Dev 18: 2404-2417.

Hartmann C, Tabin CJ (2000) Dual roles of Wnt signaling during chondrogenesis in the chicken limb. Development 127: 3141-3159.

Hartmann C, Tabin CJ (2001) Wnt-14 plays a pivotal role in inducing synovial joint formation in the developing appendicular skeleton. Cell 104: 341-351.

Hill TP, Später D, Taketo MM, Birchmeier W, Hartmann C (2005) Canonical Wnt/ $\beta$-catenin signaling prevents osteoblasts from differentiating into chondrocytes. Dev Cell 8: 727-738.

Hill TP, Taketo MM, Birchmeier W, Hartmann C (2006) Multiple roles of mesenchymal $\beta$-catenin during murine limb patterning. Development 133: 1219-1229. 
Kengaku M, Capdevila J, Rodriguez-Esteban C, De La Pena J, Johnson RL, Belmonte JC, Tabin CJ (1998) Distinct WNT pathways regulating AER formation and dorsoventral polarity in the chick limb bud. Science 280: 1274-1277.

Koyama E, Golden EB, Kirsch T, Adams SL, Chandraratna RA, Michaille JJ, Pacifici M (1999) Retinoid signaling is required for chondrocyte maturation and endochondral bone formation during limb skeletogenesis. Dev Biol 208: 375-391.

Logan CY, Nusse R (2004) The Wnt signaling pathway in development and disease. Ann Rev Cell Dev Biol 20: 781-810.

Nalin AM, Greenlee TK Jr, Sandell LJ (1995) Collagen gene expression during development of avian synovial joints: transient expression of types II and XI collagen genes in the joint capsule. Dev Dyn 203: 352-362.

Nishimura K, Solchaga LA, Caplan AI, Yoo JU, Goldberg VM, Johnstone B (1999) Chondroprogenitor cells of synovial tissue. Arthritis Rheum 42: 2631-2637.

Rodda SJ., McMahon AP (2006) Distinct roles for Hedgehog and canonical Wnt signaling in specification, differentiation and maintenance of osteoblast progenitors. Development 133: 3231-3244.

Später D, Hill TP, O'Sullivan RJ, Gruber M, Conner DA, Hartmann C (2006) Wnt9a signaling is required for joint integrity and regulation of Ihh during chondrogenesis. Development 133: 3039-3049.

Stark K, Vainio S, Vassileva G, McMahon AP (1994) Epithelial transformation of metanephric mesenchyme in the developing kidney regulated by Wnt-4. Nature 372: 679-683.

Tylzanowski P, Mebis L, Luyten FP (2006) The noggin null mouse phenotype is strain dependent and haploinsufficiency leads to skeletal defects. Dev Dyn 235: 1599-1607.

\section{Discussion with Reviewers}

I Kahn: Why does ectopic expression of Wnt9a induce gdf5 expression in (chick) limbs but not in vitro micromass cultures using limb mesenchyme?
Authors: In the previous gain-of function studies in chicken pre-cartilaginous digit anlagen were infected with Wnt9a expressing retroviral particles. Although a broader region was infected with Wnt9a retrovirus only a part of the infected area would express Gdf5 and other early joint interzone markers. These areas were always localized immediately adjacent to an uninfected cartilaginous element and probably originated from the pre-cartilaginous anlagen. Like in the in vivo situation Wnt9a in the in vitro studies inhibited inhibited chondrogenesis, but no induction of Gdf5 or any other joint interzone markers was observed. Similarly, infection of the non-chondrogenic mesenchyme never did lead to ectopic expression of Gdf5 and other joint interzone markers. This suggests that Wntsignaling is not sufficient to induce Gdf5, but probably requires additional signals originating from the adjacent cartilage element.

I Kahn: On the basis of the authors' conclusion that Wnts are not responsible for initiating joint interzone formation, could they speculate as to the identity of the factors that may perform this function?

Authors: The conclusion is that canonical Wnt-signaling alone is not required for the initiation of the joint interzone, based on the presence of molecular markers such as Gdf5, Wnt4 and Gli1. However, canonical Wnt-signaling or at least the function of $\beta$-catenin is required for the formation of a true interzone, which is characterized by the downregulation of Collagen II and Sox9. Which factors are required for the initiation of interzone markers remains an open question. The initiation of the joint interzone characterized by the expression of a variety of markers is likely to depend on signals and transcription factors involved in pre-patterning the limb along its proximo-distal axis, an aspect of limb patterning which is only poorly understood. Future experiments addressing the transcriptional regulation of the early interzone markers might lead to the identification of common elements which are responsible for the expression in the joint regions. On the basis of such common elements it might be possible to identify the key transcription factors, which are necessary and sufficient to induce restrict the expression of these markers to the joint interzone. 